\title{
The role of growth patterns, according to Kodama's classification, and lymph node status, as important prognostic factors in early gastric cancer: analysis of 412 cases
}

\author{
Luca Saragoni ${ }^{1}$, Michele Gaudio $^{1}$, Paolo Morgagni ${ }^{2}$, Secondo Folli ${ }^{2}$, Antonio Vio ${ }^{2}$, Emanuela Scarpi $^{3}$, \\ and Ariele Saragoni ${ }^{1}$ \\ ${ }^{1}$ Department of Pathology, G.B. Morgagni-L. Pierantoni hospital, Vecchiazzano (Forlì), Via Forlanini 34, 47100 Forlì, Italy \\ ${ }^{2}$ First Division of General Surgery, G.B. Morgagni-L. Pierantoni hospital, Forlì, Italy \\ ${ }^{3}$ Biostatistic Unity, Istituto Oncologico Romagnolo (IOR), Forlì, Italy
}

\begin{abstract}
Background. During the 1970s, a special type of Gastric Cancer with excellent prognosis (early gastric cancer; EGC) was identified by the Japanese Research Society for Gastric Cancer. EGC has been defined as a tumor which invades the mucosa and/or submucosa, regardless of the lymph node status. Using this definition, we identified an initial phase of tumor development which could be treated both endoscopically and surgically.

Methods. We examined 412 EGC patients, recruited between 1976 and 1999, with an average follow-up of 9 years. All tumors were classified according to the macroscopic and microscopic criteria proposed by the Japanese Society of Gastroenterological Endoscopy (JSGE) and Lauren, respectively. The infiltrative growth pattern was evaluated according to Kodama's classification. Only tumor-related death was considered as an end-point of interest for the survival analysis. Results. Submucosal tumors $(P=0.008)$, Pen A (see definition below) type disease $(P=0.0001)$, and lymph nodepositive cancers $(P=0.0002)$ were significant prognostic factors on univariate analysis. Moreover, bivariate analysis showed that the worst prognosis, in terms of survival, was for patients with nodal involvment, submucosal invasion, and node-positive and Pen-A type cancer. The abbreviation Pen, penetrating, indicates a lesion with a diameter of less than $4 \mathrm{~cm}$, which invades the submucosa diffusely. Pen A type EGC represents a subgroup of tumors which infiltrates the submucosa extensively, with nodular masses, causing the complete destruction of the muscularis mucosae.

Conclusion. In our series, Pen A type was an important prognostic factor (hazard ratio; HR, 8.32; 95\% confidence interval [CI], 3.49-19.86. For this reason, we believe it is important to evaluate the infiltration into the wall in all patients with EGC, paying particular attention to the growth pattern of the neoplasm. Moreover, submucosal Pen A type tumors had a considerably worse prognosis and this finding was reinforced when lymph node metastases coexisted. We suggest, therefore, that surgical treatment with at least a D2 lymphadenec-
\end{abstract}

Offprint requests to: L. Saragoni

Received: April 11, 2000 / Accepted: September 6, 2000 tomy is performed in all these patients, as the lesions must be considered to be advanced, no longer being EGC.

Key words EGC $\cdot$ Prognosis $\cdot$ Treatment

\section{Introduction}

During the 1970s, a special type of Gastric Cancer with excellent prognosis (early gastric cancer: EGC) was identified by the Japanese Research Society for Gastric Cancer [1,2].

EGC has been defined as a tumor which invades the mucosa and/or submucosa, regardless of the lymph node status [3-5]. Using this definition, we identified an initial phase of tumor development which could be treated both endoscopically and surgically [6-10].

The number of cases of EGC has progressively increased because of better knowledge of the problem and the consequent improvement and diffusion of endoscopic techniques [11-13]. Over the past 10 years, about $30 \%$ of all gastric cancer patients who underwent surgical treatment in our hospital had EGC [14,15].

In Japan, there are considerably more cases of EGC, now about $50 \%-60 \%$ of all gastric cancers [16]. This large number of EGCs is due both to the presence of an active mass screening program which was introduced a number of years ago [17], and to the existence of different classification systems for gastrointestinal dysplasia and gastric carcinoma in Western and Eastern countries [18-23].

Better knowledge of EGC and the improvement and diffusion of endoscopies have permitted the identification of subgroups of patients affected by EGC with a worse prognosis. These patients always require an aggressive therapeutic approach with a sub or total gastrectomy, together with an extended lymphadenectomy (D2) $[7,14,24-28]$. 
In particular, the experience we gained by evaluating 412 patients affected by EGC, with an average followup period of 9 years, has demonstrated that there is a significantly worse survival probability in node-positive submucosal and node-positive-Pen A type patients $[14,15]$. The abbreviation Pen, penetrating, indicates a lesion with a diameter of less than $4 \mathrm{~cm}$, which invades the submucosa diffusely. Pen A type EGC represents a subgroup of tumors which infiltrates the submucosa extensively, with nodular masses, causing the complete destruction of the muscularis mucosae.

All patients were recruited and treated in an Italian region where there is a high incidence of gastric cancer [29].

\section{Subjects and methods}

From 1976 to 1999, 1955 patients with gastric cancer were treated surgically at the First Department of Surgery, G.B. Morgagni-L. Pierantoni Hospital, Forlì, Italy; of these patients, 493 had EGC. The EGC/ advanced cancer ratio was $25.2 \%$. All the patients underwent a sub or total gastrectomy with a D2 lymphadenectomy. The EGCs were classified according to the macroscopic and microscopic criteria proposed by the Japanese Society of Gastroenterological Endoscopy (JSGE) [2,30] and Lauren [31], respectively. The JSGE criteria divided the EGCs into polypoid (type I), elevated (type IIa), flat (type IIb), depressed (type IIc), and ulcerated (type III).

From the histological point of view, two major categories exist, designated intestinal and diffuse by Lauren. A third group includes unclassifiable or mixed tumoral lesions. The classification of Kodama et al. [32] was used to define the extent and type of penetration of the cancer. According to this classification, super (superficial spreading) type is a tumor with a diameter of more than $4 \mathrm{~cm}$, either confined to the mucosa (super M) or with slight invasion of the submucosa (super SM); small mucosal type is a carcinoma with a diameter of less than $4 \mathrm{~cm}$, with (small mucosal SM) or without (small mucosal M) slight submucosal invasion; and Pen (penetrating) type is a lesion with a diameter of less than $4 \mathrm{~cm}$, which invades the submucosa widely. This type is further divided into two subgroups, according to the way in which the muscularis mucosae is penetrated: Pen-A type, which invades the submucosa extensively and completely destroys the muscularis mucosae, and Pen-B type, which grows infiltratively with fenestration of the muscularis mucosae.

The resected stomachs were opened along the greater curvature, pinned to a wooden plate, and fixed in $10 \%$ buffered formalin. The tumors in the surrounding gastric wall were cut into several slices, mainly parallel to the lesser curvature, at intervals of $4-5 \mathrm{~mm}$. Fivemicrometer-thick sections, embedded in paraffin, were prepared from each slide for histologic examination. All the microscopic sections, stained with hematoxylin and eosin, were examined by the same two pathologists (L.S. and M.G.) and the degree of differentiation was classified according to the outline proposed by the WHO [33].

Follow-up analysis was performed on the 412 patients who were examined. The average follow-up was 9 years (range, 1-22 years). Survival analysis started the day the EGC patients underwent surgical treatment. All the prognostic variables used in this analysis were measured at this time.

Only tumor-related death was considered as an end-point of interest for the survival analysis. Survival curves for EGC were calculated using the KaplanMeier method [34], and the difference between survival probabilities was analyzed using the log-rank test. The$\chi^{2}$ test was employed to define the association between the subgroups of pathological and clinical criteria examined. Hazard ratio (HR) evaluation, in univariate analysis, was obtained using the Cox proportional model [35]. All $P$ values were based on two-sided testing, and statistical analyses were carried out using SAS Statistical software [36].

\section{Results}

The median age of the patients was 65 years (range, 30 93 years) and the male/female ratio was $1.5: 1 ; 245$ were men $(59.5 \%)$ and 167 , women $(40.5 \%)$. About $64 \%$ of the EGCs were situated in the lower-third of the stomach and almost all cases were monofocal (90.3\%). Intramucosal and submucosal infiltration were observed with similar frequency. As expected, the most frequent types were IIc EGC (54.3\%) and small mucosal M EGC (47.8\%); according to the macroscopic (JSGE) and Kodama's classifications, respectively. Histologically, the EGC was most frequently the intestinal type (77.7\%) according to Lauren's classification. Lymph node metastases affected 55 patients $(13.3 \%)$.

The lymph node status was significantly associated with the histologic type $(P=0.001)$, infiltration of the wall $(P<0.001)$, tumor size $(P=0.001)$, Kodama's type $(P=0.001)$, and macroscopic type $(P=0.02)$. In particular, the histologically diffused type and the macroscopically depressed type ulcerated lesions (types IIc and III) and Pen A type, according to Kodama, were associated with lymph node metastases in more patients than the other subtypes (Table 1). Of the 412 patients, $284(68.9 \%)$ are still alive and $128(31.1 \%)$ died, 34 $(8.3 \%)$ as a result of the disease. The survival probability in our series was $93 \%$ at 5 years and $89 \%$ at 10 years. 
Table 1. Distribution of lymph node metastases according to Kodama [32], Lauren [31], and JSGE [2,30] classifications

\begin{tabular}{lrcc}
\hline & $\begin{array}{c}\text { Total } \\
\text { patients }\end{array}$ & $\begin{array}{c}\mathrm{N}+ \\
\text { patients }^{\mathrm{a}}\end{array}$ & $\%$ \\
\hline Growth pattern (Kodama) & & & \\
Pen A & 87 & 32 & 36.8 \\
Not Pen A & 325 & 23 & 7.1 \\
$\quad$ Small MM & 196 & 10 & 5.1 \\
$\quad$ Small Msm & 72 & 8 & 11.1 \\
$\quad$ Super M & 9 & 0 & 0 \\
$\quad$ Super Msm & 6 & 2 & 33.3 \\
$\quad$ Pen B & 41 & 3 & 7.3 \\
Histologic types (Lauren) & & & \\
Intestinal & 320 & 29 & 9.0 \\
Diffuse & 92 & 26 & 28.3 \\
Macroscopic types (JSGE) & & & \\
I & 64 & 8 & 12.5 \\
IIa & 19 & 0 & 0 \\
IIb & 19 & 0 & 0 \\
IIc & 223 & 28 & 12.6 \\
III & 86 & 19 & 22.1 \\
\hline
\end{tabular}

JSGE, Japanese Society of Gastroenterological Endoscopy

See text for definitions of growth patterns, histological types, and macroscopic types

${ }^{a} \mathrm{~N}+$, lymph node metastasis
On univariate analysis, patient age, sex, and focality were nonsignificant prognostic factors. Conversely, a significantly lower survival probability was observed for patients with submucosal tumors $(P=0.008)$, Pen A type disease $(P=0.0001)$, and node-positive cancers $(P=0.0002)$, than for the other anatomo-pathological subgroups (Table 2). In our series, in particular, the patients affected by Pen A type EGC had a death risk which was eight times higher than that in patients with non-Pen A type cancer (HR, 8.32; 95\% confidence interval (CI, 3.49-19.86). This suggests that the biological behavior of the neoplasm depends tightly on the growth pattern, according to Kodama's classification (Fig. 1). Moreover, patients with the involvement of more than three lymph nodes $(P=0.0001)$ and of distant lymph node levels $(P=0.0001)$ had a considerably worse prognosis, reflecting a more advanced stage of the disease (Figs. 2 and 3). Bivariate analyses were performed to evaluate the joint effect on survival of (a) lymph node status and Kodama's subgroups (Fig. 4), and (b) lymph node status and infiltration of the wall (Fig. 5).

\section{Discussion}

The improvement and diffusion of endoscopic techniques and a greater knowledge of the problem have led

Table 2. Univariate analysis: 5- and 10-year survival probability, hazard ratios (HRs), and their relative 95\% confidence intervals $(95 \% \mathrm{CI})$

\begin{tabular}{|c|c|c|c|c|c|c|c|}
\hline & 5-Year & $95 \% \mathrm{CI}$ & 10-Year & $95 \% \mathrm{CI}$ & Logrank & $P$ value & $\operatorname{HR}(95 \% \mathrm{CI})$ \\
\hline \multicolumn{8}{|l|}{ Depth } \\
\hline Mucosal & 96 & $93-99$ & 93 & 89-97 & & & 1.00 \\
\hline Submucosal & 88 & $83-93$ & 85 & $80-91$ & 7.05 & 0.008 & $2.60(1.25-5.4)$ \\
\hline \multicolumn{8}{|l|}{ Size } \\
\hline$<2 \mathrm{~cm}$ & 96 & $91-100$ & 94 & 89-99 & & & 1.00 \\
\hline$\geqq 2 \mathrm{~cm}$ & 88 & $82-95$ & 86 & $78-94$ & 4.85 & 0.088 & $2.43(0.94-6.2)$ \\
\hline \multicolumn{8}{|l|}{ Kodama } \\
\hline Small MM & 97 & 94-100 & 95 & $91-99$ & & & 1.00 \\
\hline Small Msm & 92 & $85-99$ & 88 & 79-96 & & & $2.50(0.88-7.13)$ \\
\hline Super M & 100 & & 100 & & & & / \\
\hline Super Msm & 100 & & 100 & & & & 1 \\
\hline Pen A & 74 & $64-85$ & 67 & $53-81$ & & & $8.32(3.49-19.86)$ \\
\hline Pen B & 97 & $92-100$ & 97 & $92-100$ & 41.64 (5 df) & 0.0001 & $0.68(0.08-5.51)$ \\
\hline \multicolumn{8}{|l|}{ Nodes } \\
\hline Negative & 94 & $91-97$ & 91 & $88-95$ & & & 1.00 \\
\hline Positive & 77 & $64-89$ & 77 & $64-89$ & 13.86 & 0.0002 & $3.57(1.74-7.32)$ \\
\hline \multicolumn{8}{|l|}{ No. nodes +} \\
\hline 0 & 94 & $91-97$ & 91 & $88-95$ & & & 1.00 \\
\hline $1-3$ & 89 & 78-99 & 89 & $78-99$ & & & $1.62(0.56-4.70)$ \\
\hline$>3$ & 42 & $13-71$ & 42 & $13-71$ & $49.80(2 \mathrm{df})$ & 0.0001 & $11.42(4.88-26.70)$ \\
\hline \multicolumn{8}{|l|}{ Node level } \\
\hline $\mathrm{N}-$ & 94 & $91-97$ & 91 & $88-95$ & & & 1.00 \\
\hline N1 & 80 & $67-94$ & 80 & $67-94$ & & & $2.79(1.20-6.51)$ \\
\hline $\mathrm{N} 2$ & 62 & $33-92$ & 62 & $33-92$ & $20.02(2 \mathrm{df})$ & 0.0001 & $6.93(2.39-20.08)$ \\
\hline
\end{tabular}

df, Degrees of freedom 

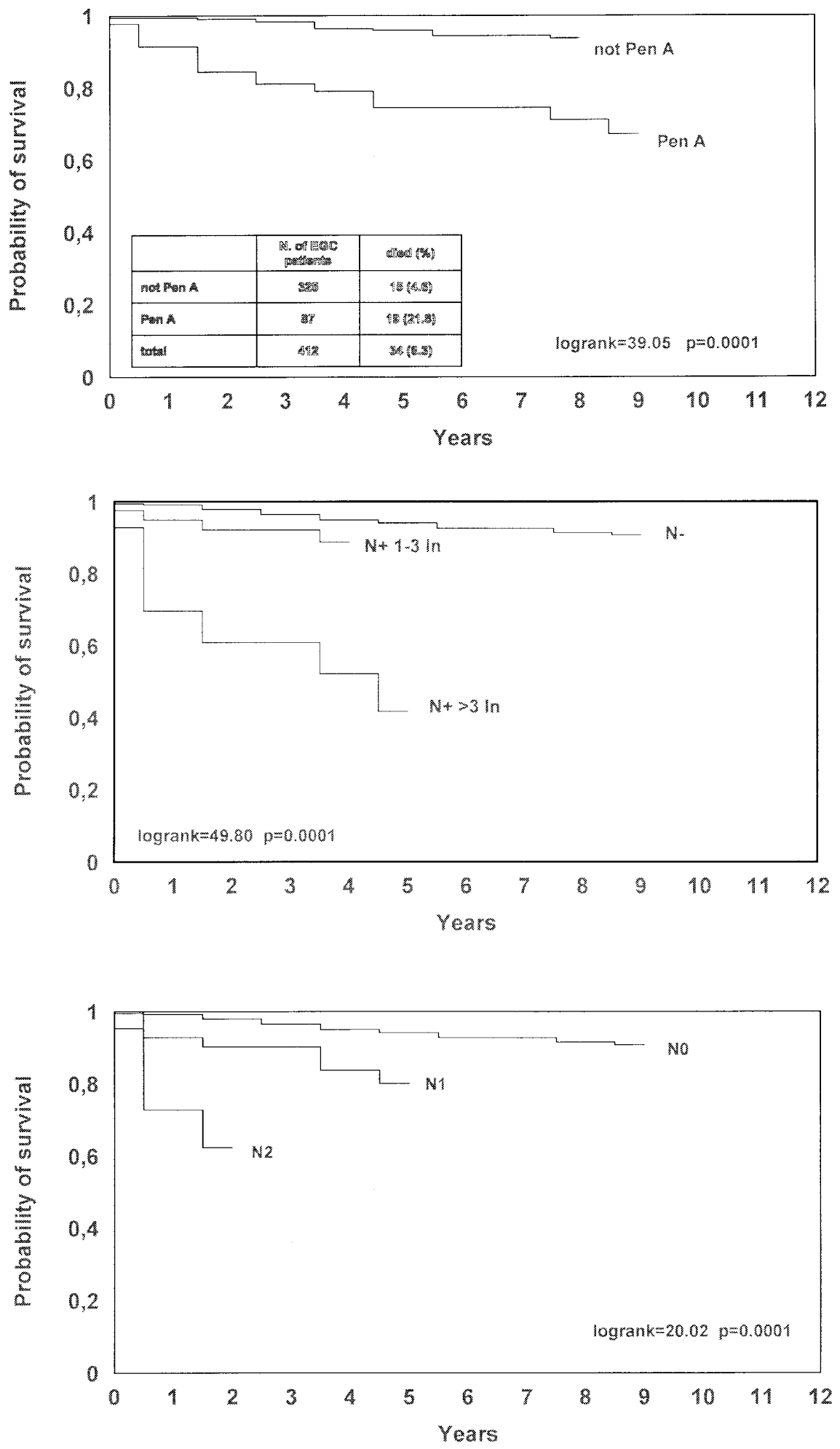

Fig. 1. Probability of survival, depending on growth pattern, according to the classification of Kodama et al. [32]. EGC, Early gastric cancer; Pen A, see text for definition

Fig. 2. Probability of survival, depending on number of lymph nodes involved. $N-$, Node-negative patients $(n=357) ; N+1-$ 3 ln patients with one to three positive nodes $(n=41) ; N+>3 \ln$ patients with more than three positive nodes $(n=14)$

Fig. 3. Probability of survival, depending on the level of lymph nodes involved. NO, Node-negative patients $(n=357) ; N 1$, level I node-positive patients $(n=42)$; $N 2$, level II node-positive patients $(n=$ 13) to a significant increase in EGC cases over the past few years. In our province, EGC represents about $30 \%$ of all resected gastric cancers [14,15]. In the 1970s, the most important prognostic factor was the depth of wall invasion. Subsequent clinical evidence, however, has consistently indicated nodal involvement as the most important prognostic parameter, so that only cancers without nodal invasion were considered as early neoplasms [8,37]. By taking this into consideration, some authors demonstrated that the disease in patients with 

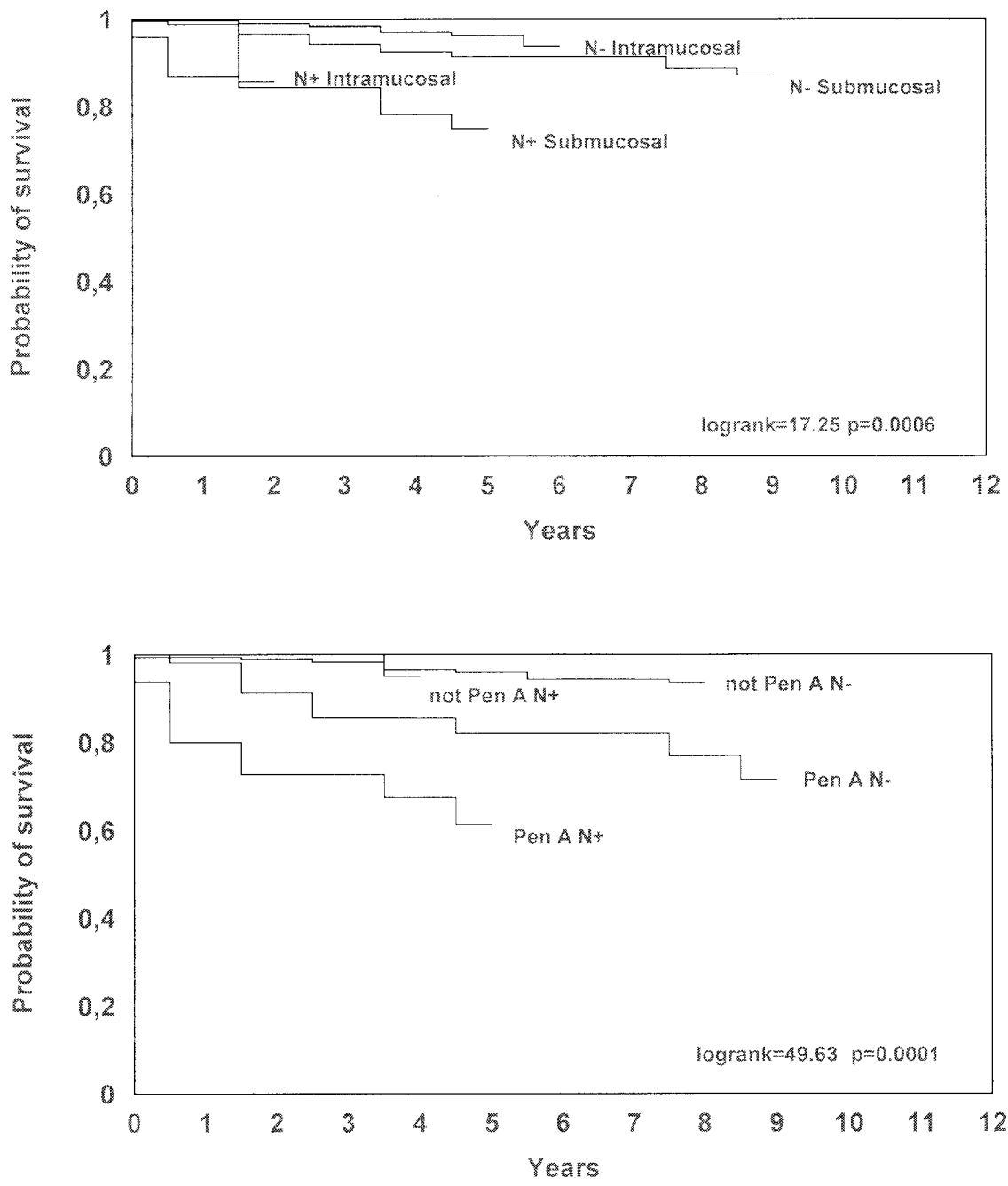

Fig. 4. Probability of survival, depending on wall and lymph node invasion: $N-$ Intramucosal, Node-negative EGC limited to mucosa ( $n=202) ; N-$ Submucosal, node-negative EGC invading submucosa $(n=155) ; N+$ Intramucosal, node-positive EGC limited to mucosa $(n=7) ; N+$ Submucosal, node-positive EGC invading submucosa $(n=48)$
Fig. 5. Probability of survival, depending on the type of growth pattern and lymph node invasion. not Pen $A N-$, Nodenegative-non Pen A patients $(n=302)$; Pen $A N-$, node-negative-Pen A patients $(n=55)$; not Pen $A N+$, node-positivenon Pen A patients $(n=23)$; Pen $A N+$, node-positive-Pen A patients $(n=32)$ gastric cancer without lymph node metastases but with invasion of the muscularis propria behaved in a similar way to the disease in conventional EGC patients $[8,38,39]$. These findings would seem to classify all nodenegative gastric cancers as Dukes' A tumors, regardless of the depth of penetration [39]. Our previous studies showed that Pen A type growth pattern and lymph node status were two important prognostic factors in EGC patients $[14,15]$. The present study, performed on a series of 412 patients recruited in a region of Italy with a high incidence of gastric cancer, confirms such results. In particular, lymph node status seems to reduce patients' survival rate when more than three lymph nodes are involved or when there are lymph node metastases in the distal levels, according to the Japanese classification. Our results reinforce the role of Pen A type EGC, according to Kodama's criteria, as a significant prognostic factor, in both node-negative and node-positive EGC. Moreover, in our series, non-Pen A types had a better prognosis than node-negative Pen A EGC, even when associated with the presence of lymph node metastases. The presence of nodal involvement and a Pen A type growth pattern reduced the survival rate of our patients (61\% at 5 years). Such tumors, although they are classified as EGC according to the Japanese Research Society for Gastric Cancer definition, have a significantly worse prognosis and, in our opinion, must be considered as advanced lesions. Therefore, this knowledge suggests that we must pay particular attention to these tumors, in order to perform the best surgical treatment, and this knowledge is worthy of consideration in subsequent studies, eventually to modify the definition of EGC.

Apart from our results, few reports have suggested modifying the definition of EGC, by identifying subgroups of patients with nodal metastases, characterized by worse prognosis $[8,38,39]$. According to these authors, infiltration into the wall was not as important as lymph node status for establishing the biological behavior and prognosis of the tumor. Some studies, therefore, tried to introduce Duke's classification for EGC [39]. Our conclusion provides a new contribution to the gen- 
eral knowledge of EGC, because of the identification of a new important prognostic factor, which is represented by Kodama's classification, which takes into consideration the extent and type of infiltration into the gastric wall.

In particular, submucosal Pen A type tumors had a significantly worse prognosis in our series and this finding was reinforced when lymph node metastases coexisted.

In our patients, however, Pen A type was a significant prognostic factor (HR, 8.32; 95\% CI, 3.49-19.86). For this reason, we believe it is important to evaluate infiltration into the wall in all EGC patients, paying particular attention to the growth pattern of the neoplasm. These results are in agreement with our previous reports $[14,15]$, even though there was a smaller number of patients in the previous reports.

In conclusion, we consider Dukes' classification to be well applied to gastric cancer as a simple and useful staging system [39] and wish to emphasise the important role of growth pattern, according to Kodama's classification, in identifying a subgroup of patients affected by a more aggressive type of EGC, with clinical behavior similar to that of an advanced lesion. Moreover, in these patients, we suggest performing surgical treatment, which includes at least D2 extended lymphadenectomy, as several Japanese authors have previously proposed.

\section{References}

1. Japanese Research Society for Gastric Cancer. The general rules for the gastric cancer study in surgery and pathology. Jpn J Surg 1981;11:127-39.

2. Murakami T. Pathomorphological diagnosis, definition and gross classification of early gastric cancer. Gann Monograph Cancer Res 1971;11:53-66.

3. Fenoglio CC, Lantz P, Listrom MB, Davis M, Rilke FO. Gastrointestinal pathology. 1st ed. New York: Raven; 1989.

4. Folli S, Dente M, Dell'Amore D, Amadori D, Vio A. Il carcinoma gastrico superficiale. Esperienza clinica in un'area ad alto rischio. Monografia della Collana di Oncologia chirurgica. Forlì: Valbonesi; 1992.

5. Maehara Y, Orita H, Okuyama T, Moriguchi S, Tsujitani S, Corenaga D, Sugimachi K. Predictors of lymph node metastasis in early gastric cancer. Br J Surg 1992;79:245-7.

6. El-Zimaity HM, Ota H. Endoscopic resection for early gastric cancer: possibilities and limitations. J Clin Gastroenterol 1999; 29:5-6.

7. Harrison JD, Fielding JWL. Prognostic factors for gastric cancer influencing clinical practice. World J Surg 1995;19:496-500.

8. Inoue K, Tobe T, Kan N, Nio Y, Sakai M, Takeuchi E, et al. Problems in the definition and treatment of early gastric cancer. Br J Surg 1991;78:818-21.

9. Sano T, Kobori O, Muto T. Lymph node metastasis from gastric cancer: endoscopic resection of tumour. Br J Surg 1992;79:241-4.

10. Yokota T, Saito T, Teshima S, Kikuchi S, Kunii Y, Yamauchi H. Lymph node metastasis in early gastric cancer: how can surgeons perform limited surgery? Int Surg 1998;83:287-90.

11. Chang YK, Park SW, Park SJ, Hur B, Koo JY. A clinicopathological study on the endoscopic diagnosis of early gastric cancer.
Third International gastric cancer congress, Seoul (Korea), April 27-30. Bologna: Monduzzi Editore; 1999.

12. Yoshida S, Yamaguchi H, Tajiri H, Saito D. Diagnosis of early gastric cancer seen as less malignant endoscopically. Jpn J Clin Oncol 1984;14:225-41.

13. Yoshida S, Yoshimori $M$, Hirashima $T$, Yamaguchi $H$. Nonulcerative lesions detected by endoscopy as an early expression of gastric malignancy. Jpn J Clin Oncol 1981;11:495-506.

14. Folli S, Dente M, Dell'Amore D, Gaudio M, Nanni O, Saragoni L, Vio A. Early gastric cancer: prognostic factors in 223 patients. Br J Surg 1995;82:952-6.

15. Saragoni L, Gaudio M, Vio A, Folli S, Nanni O, Saragoni A. Early gastric cancer in the province of Forlì: follow-up of 337 patients in a high risk region for gastric cancer. Oncol Rep 1998;5:945-8.

16. Sano T, Sasako M, Kinoshita T, Maruyama K. Recurrence of early gastric cancer. Follow-up of 1475 patients and review of Japanese literature. Cancer 1993;72:3174-8.

17. Aochi K. Trends in stomach cancer mortality in Japanese women: an evaluation of prevention programs. Third International gastric cancer congress, Seoul (Korea), April 27-30, 1999. Bologna: Monduzzi Editore; 1999.

18. Genta RM, Rugge M. Gastric precancerous lesions: heading for an international consensus. Gut 1999;(Suppl 1):5-8.

19. Kim YI. Definition, classification and epidemiology of dysplasia. Third international gastric cancer congress, Seoul (Korea), April 27-30, 1999. Bologna: Monduzzi Editore; 1999.

20. Lauwers GY, Shimizu M, Correa P, Riddell RH, Kato Y, Lewin $\mathrm{KJ}$, et al. Evaluation of gastric biopsies for neoplasia: differences between Japanese and Western pathologists. Am J Surg Pathol 1999;23:511-8.

21. Lewin KJ. Nomenclature problems of gastrointestinal epithelial neoplasia. Am J Surg Pathol 1998;22:1043-7.

22. Riddel RH, Iwafuchi M. Problems arising from Eastern and Western classification systems for gastrointestinal dysplasia and carcinoma: are they resolvable? Histopathology 1998;33:197202.

23. Rugge M, Farinati F, Di Mario F, Baffe R, Valiante F, Cardin F. Gastric epithelial dysplasia: a prospective multicenter follow-up study from the interdisciplinary group on gastric dysplasia. Hum Pathol 1991;22:1002-8.

24. De Manzoni G, Verlato G, Guglielmi A, Laterza E, Genna M, Cordiano C. Prognostic significance of lymph node dissection in gastric cancer. Br J Surg 1996;86:1604-7.

25. Pinto E, Roviello F, De Stefano A, Vindigni C. Early gastric cancer: report on 142 cases observed over 13 years. Jpn J Clin Oncol 1994;24:12-19.

26. Roviello F, De Manzoni G, Morgagni P, Marrelli D, Di Leo A, Saragoni L, et al. A clinicopathological analysis of tumour recurrence following curative surgery for gastric cancer. The results of an Italian multicenter study. Third International gastric cancer congress, Seoul (Korea), April 27-30, 1999. Bologna: Monduzzi Editore; 1999.

27. Sendler A, Dittler HJ, Feussner H, Nekarda H, Bollschweiler E, Fink U, et al. Preoperative staging of gastric cancer as precondition for multimodal treatment. World J Surg 1995;19:501-8.

28. Siewert JR, Fink U, Sendler A, Becker K, Bottcher K, Feldmann HJ, et al. Current problems in surgery. Gastric Cancer 1997; 34:835-942.

29. International agency for research on cancer, World Health Organization and International Association of cancer Registries. Cancer incidence in five continents. vol. VII. Lyon: IARC Scientific Publications N.143; 1997.

30. Ohta H, Noguchi Y, Takagi K, Nishi M, Kajitani T, Kato Y. Early gastric carcinoma with special reference to macroscopic classification. Cancer 1987;60:1099-106.

31. Lauren P. The two main histological types of gastric carcinoma: an attempt to reach a histoclinical classification. Acta Pathol Microbiol Scand 1965;64:31-49. 
32. Kodama Y, Inokuchi K, Soejima K, Matsusaka K, Okamura T. Growth patterns and prognosis in early gastric cancer. Superficially spreading and penetrating growth types. Cancer 1983;51: 320-6.

33. World Health Organization. Histological typing of oesophageal and gastric tumors. 2nd ed. In: Watanabe H, Jass JR, Sobin LH, editors. International histological classification of tumors, N.18. Berlin Heidelberg New York Tokyo: Springer-Verlag; 1990. pp. 20-3.

34. Kaplan EL, Meier P. Non parametric estimation from incomplete observations. J Am Stat Assoc 1958;53:457-81.

35. Cox DR. Regression models and life tables. J R Stat Soc 1972;34:187-220.
36. SAS Institute. SAS/STAT User's guide, version 6. 4th ed., vol. 1. Cary, NC: SAS Institute; 1989. p. 943.

37. Kim JP, Hur YS, Yang HK. Lymph node metastasis as a significant prognostic factor in early gastric cancer: analysis of 1136 early cancers. Ann Surg Oncol 1995;2:308-13.

38. Abe S, Yoshimura $\mathrm{H}$, Nagaoka $\mathrm{S}$, Monden $\mathrm{N}$, Kinugasa $\mathrm{S}$, Nagasue N, et al. Long-term results of operation for carcinoma of the stomach in T1/T2 stages: critical evaluation of the concept of early carcinoma of the stomach. J Am Coll Surg 1995;181:38996.

39. Adachi Y, Masafuni I, Seigo K, Masaki M, Yoshihiko M, Keizo S. Dukes' A tumor: new criteria for early gastric cancer. Oncol Rep 1997;4:1235-7. 